\title{
ЭФФЕКТИВНОСТЬ ВНЕДРЕНИЯ СОВРЕМЕННЫХ ТЕХНОЛОГИЙ В БИЗНЕС-ПРОЦЕССЫ ОРГАНИЗАЦИИ
}

\author{
(c) 2021 Оганнисян Корюн Агванович \\ Студент 4-го курса, Факультет «Высшая школа управления» \\ Финансовый университет при Правительстве Российской Федерации, Россия, Москва \\ E-mail: ogannisyan.koryun@yandex.ru
}

Научный руководитель Удальцова Наталья Леонидовна

кандидат экономических наук, доцент департамента Менеджмента и инноваций, Факультет «Высшая школа управления»

Финансовый университет при Правительстве Российской Федерации, Россия, Москва

E-mail: udaltsova.nl@yandex.ru

Становление цифровой экономики все больше оказывает влияние на современные компании. Возникает необходимость цифровой трансформации бизнеса и его оптимизации под условия сегодняшнего дня. Статья направлена на анализ понятий цифровой трансформации и цифровой экономики, а также выявление положительных эффектов от внедрения различных современных технологий в бизнес-процессы организаций.

Ключевые слова: цифровая трансформация, инновации, цифровая экономика, бизнес-процессы, технологии.

\section{Введение}

Технологические процессы все более плотно проникают во все сферы современной жизни. Трудно себе представить сегодня успешный бизнес, который может обойтись без технических средств. Все большее развитие получает процесс оцифровки бизнес-процессов, например CRM, ERP, документооборот, управление персоналом.

Таким образом, мы сталкиваемся с цифровой трансформацией, которая представляет собой непрерывный процесс внедрения технологических средств и цифровых технологий во все сферы ведения бизнеса. Наличие в определении слова «трансформация» подчеркивает, что влияние процесса внедрения происходит не в отдельном сегменте, а касается каждого аспекта бизнеса. Оно влияет на дальнейшую стратегию, процесс производства, конечный продукт, политику менеджмента и маркетинга, коммуникационную составляющую и т.д. Поэтому цифровую трансформацию нельзя ассоциировать лишь с переводом данных компании в цифровой формат.

\section{Основная часть}

Несомненно, цифровизация данных оказала значительное влияния на появления процесса цифровой трансформации, так как с течением времени для эффективного ведения бизнеса этого стало недостаточно. Возникла необходимость качественного управления оцифрованной информацией, для чего потребовалось специальное программное обеспечение, позволяющее эти данные обрабатывать, синхронизировать и применять для нужд компаний, например изучения конкретных показателей на основе имеющихся данных, составления отчетов о сделанной работе с целью формирования как долгосрочной, так и краткосрочной стратегий. Дальнейшее развитие этого направления показало, что применения программного обеспечения так же становится недостаточно, что привело к необходимости внедрения изменений непосредственно в бизнес-процессы [5]. Рассмотрим объекты digital-трансформации (рис. 1).

Основные процессы, которые затрагивает цифровая трансформация можно разделить на несколько блоков. Рассмотрим каждый из них.

1. Работа с данными. Каждая компания сталкивается с ежедневным накоплением информации, это так называемые Big Data. Поэтому существует необходимость обработки и анализа этих данных, а также улучшения продуктов на основании такого анализа.

2. Коммуникации с клиентом. На сегод- 


\section{Объекты digital-трансформации}

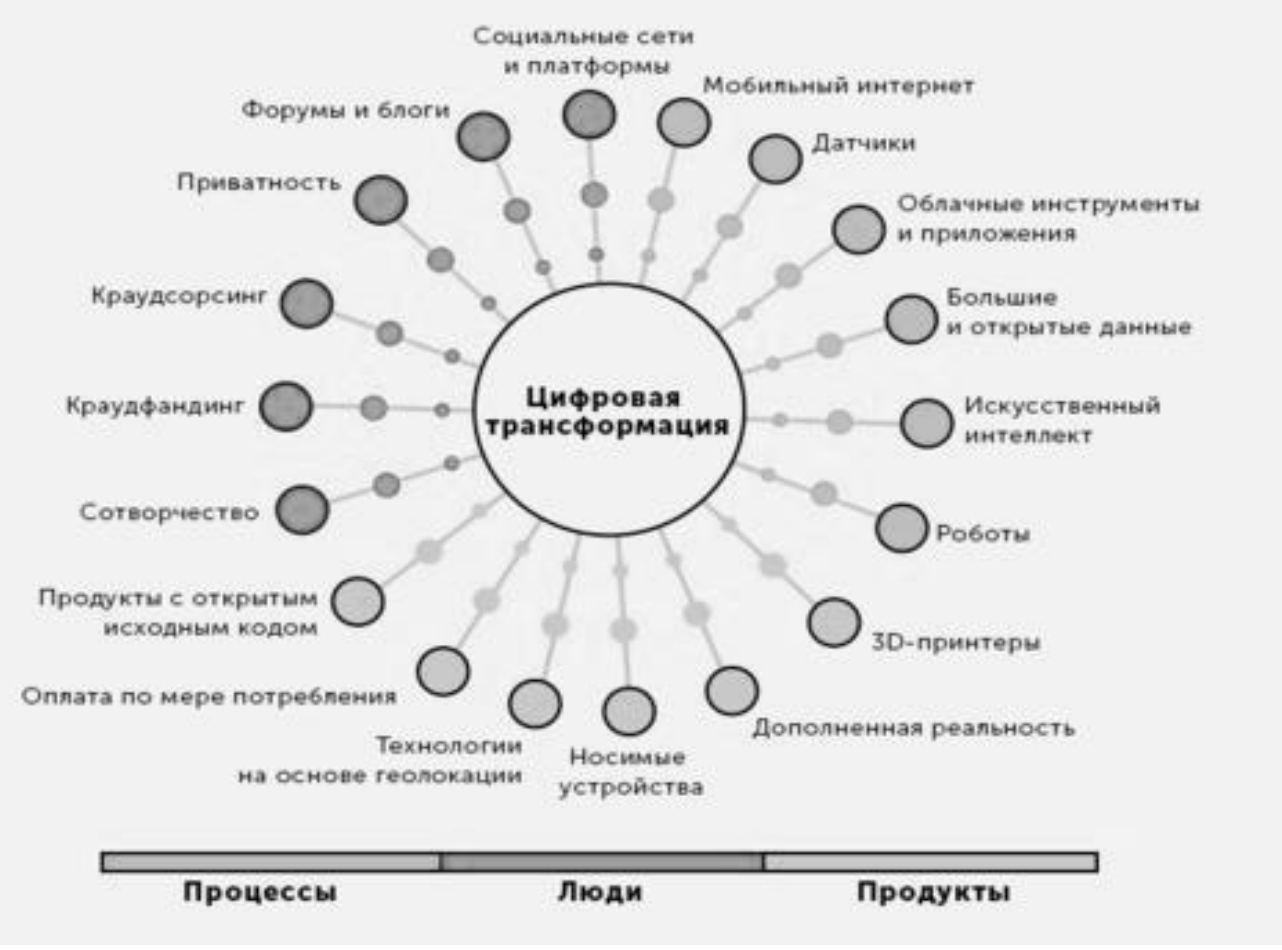

Puc. 1. Объекты digital-трансформации.

Источник: Персональный журнал руководителя «Генеральный директор» [4]

няшний день эффективное управление компании должно опираться, в том числе, и на лояльность клиентов, в связи с этим, цифровые инструменты помогают быть на связи с клиентами постоянно, а не только в отведенное рабочее время. Это позволяет непрерывно принимать заказы, обеспечивать процесс коммуникации, подготавливать персональные рекламные предложения.

3. Партнерское взаимодействие. В этом сегменте так же решающим фактором выступает время, поэтому с помощью средств цифровой трансформации общение может быть бесперебойным, а отправка сообщений мгновенной. Скорость обмена информацией напрямую будет влиять на качество бизнес-процессов.

4. Ориентация на инновации в построении рабочего процесса. Применение CRM-систем на сегодняшний день является неотъемлемой частью компаний, которые следят за качеством бизнес-процессов, однако, прогрессивные менеджеры уже постепенно переходят на новый уровень, изучая такие системы управления как Lean, Kanban, Agile и SCRUM. Причем, каждая из этих систем имеет собственное программное обеспечение [3].

Таким образом, процесс цифровой транс- формации затрагивает не только техническую часть ведения бизнеса. Это комплексная система, которая затрагивает весь рабочий процесс, включая работу с клиентами и внутри коллектива, работу с контрагентами, внедрение инновационной системы менеджмента и маркетинга, разработку и применение новых продуктов [4].

Исторически появление термина «цифровая трансформация относится к концу XX века. Как уже было отмечено ранее, цифровые технологии постепенно стали выходить за рамки программного обеспечения и стали влиять на форму ведения бизнеса. Цифровая трансформация - это явление, которое активно развивается в бизнессреде. Его нельзя игнорировать, поэтому, компании, которые смогут адаптироваться к условиям, которые диктует современный бизнес, будут наиболее конкурентоспособные.

Цифровая трансформация может оказывать как положительное, так и отрицательное влияние на деятельность компании. Это, в первую очередь, означает, что в огромном потоке данных и наличии разнообразных каналов впереди будет та компания, которая сможет наиболее быстро подключиться к глобальному процессу цифровой трансформации. Существует множество определений понятия «цифровая транс- 
формация». Рассмотрим некоторые из них, которые были представлены экспертами в сферах менеджмента и экономики.

Простейшее определение данного понятия предлагает Boston Consulting Groop (BCG). По их мнению, цифровая трансформация представляет собой наиболее полное использование технологического оснащения во всех сферах ведения бизнеса компании [1]. В данной точке зрения упор сделан на масштабы внедрения, а не масштабы изменений.

Мнение агентства Rufus Leonard описывает понятие цифровой трансформации как еще более обширной категории, которая затрагивает не только внутреннюю среду компании, но и внешнюю. Основными движущими силами трансформации агентство выделяет: изменения запросов пользователей, развития технологий и усиления конкуренции [2].

Ряд авторов обращает внимания на результаты данного процесса. Так, Глобальный центр по цифровой трансформации бизнеса дал следующее определение цифровой трансформации: “Это мероприятия по внедрению цифровых технологий в существующие бизнес-модели для достижения результата, который можно определить в количественном выражении" [7]. В данном случае можно видеть упор на рост производительности. Не обошли вниманием и деловую культуру. Так, В.Рыжков дал следующее определение: “Цифровая трансформация есть смена бизнес-мышления в динамичных условиях цифровой экономики, где главные стимулы развития - это потребители и меняющаяся культура коммуникаций» [11].

Рассмотрев ряд мнений на понятие цифровой трансформации, следует отметить, что наиболее полной, по нашему мнению, будет версия определения, предложенная аналитиком компании Agile Elephant Д. Терраром. Он предположил, что цифровая трансформация представляет собой непрерывный процесс перехода организации к новым способам мышления и построение всей системы организации работы предприятия с применением различного рода цифровых технологий. Таким образом, трансформация должна коснуться всех областей, а именно: стиля руководства, системы поощрений, ввода инновационных продуктов в работу, принятия новых бизнес-моделей для организации эффективной работы как среди сотрудников, так и для взаимодействия компании с контрагентами [18].
K рассмотренным определениям следует добавить, что производимые изменения во внутренней и внешней среде компании должны положительно сказаться на ее деятельности. Цифровая трансформация как процесс должен быть стратегически управляем, так как в сегодняшних условиях компании должны быть готовы столкнуться с вызовами цифровой экономики, ведь речь идет не просто об автоматизации, а о сложном процессе с точки зрения его реализации. Что касается определения цифровой экономики, то в мировой практике по сей день не сложилось четкого понимания данного термина. Большая часть зарубежных специалистов, рассматривая цифровую экономику, акцентируют внимание на внедрение технологий в процесс взаимодействия экономических субъектов.

Некоторые авторы считали цифровую экономику новым режимом экономики, в центре которой выступает информация и технологии [10]. Другие определяют ее как рынки нового поколения, построенные на цифровых технологиях [17]. Третьи же представляли цифровую экономику как активность, возникающую через сетевое взаимодействие людей и предприятий с использованием технических устройств путем обмена данных и организации различных процессов [15]. Развитие цифровой экономики стимулирует экономический рост, так как возрастает конкуренция, компании становятся более привлекательны в инвестиционном плане, а это, в свою очередь, улучшает уровень услуг, способствует увеличению возможностей выбора для потребителя, а также влияет на создание дополнительных рабочих мест [16].

Общепринятого понятия цифровой экономики пока нет и в России, однако, можно выделить ключевые характеристики, которые соответствуют данному явлению. Согласно материалам Послания Президента Российской Федерации Федеральному Собранию от 1 декабря 2016 г, цифровая экономика представляет собой экономику новейшего технологического поколения [8]. А если рассматривать данное понятие согласно Стратегии развития информационного общества РФ на 2017-2030 гг., то цифровая экономика будет представлять собой организацию хозяйственной деятельности, основу которой составляет большое количество данных в цифровом виде. Обработка таких данных путем применения технических средств позволяют добиться более высоких результатов, нежели при 
традиционной форме ведения бизнеса. Внедрение цифровых технологий позволяет повышать эффективность производства, ускорять процессы на определенных видах оборудования, оптимизировать процессы по хранению, транспортировке, доставке товаров различного характера [12].

Рассмотрев возможные варианты определений, можем выделить главные составляющие рассматриваемого понятия. Цифровая экономика является деятельностью по созданию, использованию и распространению цифровых технологии, которые представляют собой способы сбора, хранения, анализа, поиска и передачи данных в электронном виде. Таким образом, несмотря на то, что применение цифровых технологий во всех сферах уже является не новым направлением, как на государственном, так и на корпоративном уровне, следует отметить наступление нового этапа в экономике всего мира, так называемой цифровой экономики. Данное явление затрагивает все сферы жизнедеятельности, а предприятия, в свою очередь, все чаще становятся на путь применения цифровой трансформации.

Следует отметить, что еще не успели обрести свои конечные определения новые понятия, рассмотренные в данном исследовании, однако их развитие уже идет весьма быстрыми темпами. Но на сегодняшний день еще отсутствуют полноценные механизмы регулирования и правовая база по данным направлениям. А это, безусловно, будет сдерживать темп развития как цифровой экономики, так и позитивных эффектов для бизнеса, связанных с ней. Без применения современных технологий невозможно представить эффективное развитие и масштабирование бизнеса любой компании. Растущая конкуренция на рынке, снижение лояльности клиентов подталкивают к внедрению, либо совершенствованию уже имеющихся технологий в процесс организации хозяйственной деятельности компании. Современные технологии, которые грамотно подобраны под вид бизнеса помогают расширить клиентскую базу, открыть новые потенциалы в работе, получить возможности, которых раньше еще не было. Технологические и цифровые инструменты, применяемые при цифровой трансформации бизнеса, позволяют полностью контролировать бизнеспроцессы, протекающие в организации, а также предоставлять индивидуальный подход, который так ценят клиенты. Кроме того, применение современных технологий позволит решить ряд задач:

1. Выстроить грамотную систему коммуникации компании с клиентом;

2. Провести оптимизацию издержек;

3. Увеличить продуктивность работы персонала;

4. Автоматизировать ряд бизнес-процессов;

5. Обрести конкурентное преимущество на рынке;

6. Вывести маркетинг на новый уровень;

7. Разработать стратегию развития и роста компании.

Любое технологическое новшество направлено на создание вокруг человека «умного пространства». Мы привыкли, что вокруг нас множество гаджетов, систем, сервисов, которые направлены на то, чтобы упростить привычные вещи, сэкономить время, сделать более удобными повседневные дела. Рассмотрим некоторые выбранные примеры современных технологий, способных оказать положительный эффект на бизнес-процессы организации (Таблица 1).

Таблица 1. Примеры современных технологий, способных оказать положительный эффект на бизнес-процессы организации

\begin{tabular}{|c|l|l|}
\hline № & \multicolumn{1}{|c|}{ Наименование } & \multicolumn{1}{c|}{ Описание } \\
\hline 1 & $\begin{array}{l}\text { Industrial Internet of } \\
\text { Things (IIоT)- ин- } \\
\text { тернет вещей. }\end{array}$ & $\begin{array}{l}\text { Работает на основе совокупности компьютерных сетей и объектов со встро- } \\
\text { енными датчиками. Удаленно может проводиться контроль и управление в } \\
\text { автоматизированном режиме. Принцип работы состоит в размещении датчи- } \\
\text { ков на ключевые механизмы, посредством которых будут собираться данные о } \\
\text { состоянии предприятия, что позволит применить их для анализа и оптимиза- } \\
\text { ции бизнес-процессов [9]. }\end{array}$ \\
\hline 2 & Блокчейн & $\begin{array}{l}\text { Представляет собой технологию, позволяющую оптимизировать процесс хране- } \\
\text { ния, передачи и производства данных. Применительно на практике, блокчейн } \\
\text { представляет собой ряд блоков трансакций, позволяющих отслеживать все дей- } \\
\text { ствия в той или иной системе. Например, улучшение системы здравооранения } \\
\text { и оказания услуг путем изучения данных пациентов, полученных из разных } \\
\text { источников (медицинские карты, нательные личные устройства, клинические } \\
\text { испытания и т.д.). по подсчетам специалистов, применение данной технологии } \\
\text { на производстве позволит снизить затраты на 10-20\% [13]. }\end{array}$ \\
\hline
\end{tabular}




\begin{tabular}{|c|c|c|}
\hline 3 & NX-система & $\begin{array}{l}\text { NX-система. Данное нововведение позволит автоматизировать процесс про- } \\
\text { ектирования изделий. Кроме того, система предлагает возможности проек- } \\
\text { тирования части изделия, изменение его дизайна, проведение инженерного } \\
\text { анализа, а также программирование оборудование, на котором изделие будет } \\
\text { произведено. Разработчиком выступает компания Siemens. Таким образом, } \\
\text { внедрив данную технологию можно значительно сократить время на произ- } \\
\text { водственный процесс, а также повысить качество продукции, так как система } \\
\text { проводит инженерный анализ и настраивает оборудование под производство } \\
\text { определенного вида продукции. Однако, следует помнить, что сбой системы } \\
\text { может повлечь значительные убытки [14]. }\end{array}$ \\
\hline 4 & CRM-система & $\begin{array}{l}\text { Неотъемлемая часть организации работы с клиентами организации, которая } \\
\text { намерена оптимизировать бизнес-процессы. } \\
\text { Данная система построена на синхронизации разрозненных данных о клиен- } \\
\text { тах, позволяет выбрать наиболее оптимальную стратегию работы с клиентами. } \\
\text { Автоматизация системы позволяет хранить все документы в электронном виде, } \\
\text { а наличие полной информации о клиенте может быть полезным при ориента- } \\
\text { ции маркетинговой кампании на определенного клиента. }\end{array}$ \\
\hline 5 & $\begin{array}{l}\text { Облачные техноло- } \\
\text { гии }\end{array}$ & $\begin{array}{l}\text { Облачные технологии позволяют хранить большие объемы данных все цифро- } \\
\text { вого носителя и предоставляют доступ к ним в любое удобное время. }\end{array}$ \\
\hline 6 & $\begin{array}{l}\text { SCM-система } \\
\text { управления цепями } \\
\text { поставок }\end{array}$ & $\begin{array}{l}\text { Данная система позволяет оптимизировать логистику компании. Она представ- } \\
\text { ляет собой программное обеспечение, которое позволяет прогнозировать объе- } \\
\text { мы продаж товаров, необходимый гарантийный запас продукции, планировать } \\
\text { объемы поставок продукции, а также учитывать факторы, влияющие на прода- } \\
\text { жи. Применение данной технологии способно повлиять не только на управле- } \\
\text { ние и оптимизацию цепей поставок, но и на такие важные бизнес-процессы на } \\
\text { предприятии как: срок вывода продукта на рынок за счет оптимизации склад- } \\
\text { ской политики, то есть сокращения простоев на складах или предотвращение } \\
\text { ситуаций, при которых производственных запасов на предприятии не хватает } \\
\text { для производства продукции. Также она влияет на сокращение излишков про- } \\
\text { дукции на складе готовой продукции, например предприятие закупило про-- } \\
\text { дукцию, которую доставили ровно в срок, соответственно, производственный } \\
\text { процесс происходит без отклонений, и заказчик получает продукцию ровно } \\
\text { в запланированный срок. Данная технология позволяет сократить затраты на } \\
\text { хранение запасов на 20-50\% [6]. }\end{array}$ \\
\hline
\end{tabular}

Источник: разработано автором по данным [6, 9, 13, 14]

\section{Заключение}

Таким образом, рассмотрен ряд современных цифровых технологий, которые способны улучшать протекающие на предприятии бизнеспроцессы, а также определили их положительный эффект при внедрении на предприятие. Закономерность заключается в том, что все технологии связаны между собой, так как каждая из них способна повлиять на любой бизнес-процесс, например, цифровые технологии, применяемые в процессах, отвечающих за качество продукции, влияют на срок вывода продукции на рынок, эффективность производства и логистику.

Современные технологии помогают управлять бизнесом, удерживать клиентов и привлекать новых покупателей, предоставлять необходимую информацию в понятном виде. Бизнес трансформируется и плотно связывается с новыми технологиями, этот процесс неизбежен и необходим при условии, что бизнес желает развиваться и расти.

\section{Библиографический список}

1. Банке Барт. Аналитический отчет BCG. Vlast.kz. [Электронный ресурс].- Режим доступа: URL: https://vlast. kz/corporation/24539-cifrovizacia-biznesa.html

2. Вьюгина Д. М. Цифровые стратегии медиабизнеса в условиях изменяющегося медиапотребления // Медиаскоп. - 2016. - № 4. / [Электронный ресурс]. - Режим доступа: URL: http://www.mediascope.ru/2233

3. Головенчик, Г.Г. Теоретические подходы к определению понятия «цифровая экономика» / Г. Г. Головенчик // Наука и инновации. - 2019.- № 2.- С. 40-45 
4. Ипатова Анна. Диджитал-трансформация в России и мире // Генеральный Директор.- 2020. / [Электронный ресурс].- Режим доступа: URL: https://www.gd.ru/articles/10442-didjital-transformatsiya

5. Кузовкова, Т. А. Обоснование эволюции критериев цифрового развития экономики и общества / Т. А. Кузовкова, Е. Г. Кухаренко, Т. Ю. Салютина // Экономика и качество систем связи. - 2019. - № 2.- С. 13-20

6. Майоров, М.Цифровые технологии в производстве / М. Майоров // Бизнес-Журнал. - 2019, [Электронный pecypc]. - Режим доступа: URL: http://business71.ru/?p= modules \&modname=news \&r=fullnews \&id=31258

7. Отчет Глобального центра по цифровой трансформации бизнеса: Digital Vortex. Digital Vortex: How Digital Disruption Is Redefining Industries (cisco.com) How Digital Disruption Is Redefining Industries, Digital Vortex Global Center for Digital Business Transformation (studylib.net)

8. Послание Президента Российской Федерации от 01.12.2016 г. б/н (О положении в стране и основных направлениях внутренней и внешней политики государства) [Электронный ресурс].- Режим доступа: URL: http://www.kremlin.ru/acts/bank/41550

9. Промышленный интернет вещей // Tadviser: государство, бизнес, ИТ.- 2018. [Электронный ресурс].- Режим доступа: URL: http://www.tadviser.ru/index.php/IIoT___Industrial_Internet_of_Things

10. Развитие цифровой экономики в России // Всемирный банк.-2016. / [Электронный ресурс].- Режим доступа: URL:<http://www.vsemirnyjbank.org/ru/events/2016/12/20/developing-thedigital-economy-in-russiainternational-seminar-1>

11. Рыжков В. Что такое digital-трансформация? Komanda-a.pro. [Электронный ресурс].- Режим доступа: URL: http://komanda-a.pro/blog/digital-transformation

12. Указ Президента Российской Федерации от 09.05.2017 г. № 203 О Стратегии развития информационного общества в Российской Федерации на 2017-2030 годы. [Электронный ресурс]. - Режим доступа: URL http:// www.kremlin.ru/acts/bank/41919

13. Хассе, Ф. Блокчейн - новые возможности для производителей и потребителей электроэнергии? / Ф. Хассе // Обзор мировой электроэнергетики, подготовленный PWC.-2018. С. 16-18.

14. Цифровое предприятие: трансформация в новую реальность / В. И. Ананьин [и др.]// Бизнес-информатика.2018 - № 2(44) - C. 45-52.

15. Deloitte (2019). What is Digital Economy? [Электронный ресурс].- Режим доступа: URL: <https://www2. deloitte.com/mt/en/pages/technology/articles/mt-what-is-digital-economy.html>

16. European Commission (2018) Digital Economy. [Электронный ресурс].- Режим доступа: URL: <https:// ec.europa.eu/jrc/en/research-topic/digital-economy>

17. Fayyaz S. (2018). A review on measuring digital trade \& e-commerce as new economic statistics products. The 16th Conference of IAOS. [Электронный ресурс].- Режим доступа: URL: <http://www.oecd.org/iaos2018/ programme/IAOS-OECD2018_Fayyaz.pdf>

18. Terrar David What is Digital Transformation? Theagileelephant.com. [Электронный ресурс].- Режим доступа: URL: http://www.theagileelephant.com/what-is-digital-transformation 